\title{
Control of intestinal Nod2-mediated peptidoglycan recognition by epithelium-associated lymphocytes
}

\author{
CU Duerr ${ }^{1,2}$, NH Salzman ${ }^{3}$, A Dupont ${ }^{1}$, A Szabo $^{3}$, BH Normark ${ }^{2,4}$, S Normark $^{2,4}$, RM Locksley $^{5}$, \\ P Mellroth $^{2}$ and MW Hornef ${ }^{1}$
}

Innate immune recognition of the bacterial cell wall constituent peptidoglycan by the cytosolic nucleotide-binding oligomerization domain 2 (Nod2) receptor has a pivotal role in the maintenance of intestinal mucosal homeostasis. Whereas peptidoglycan cleavage by gut-derived lysozyme preserves the recognition motif, the $\mathrm{N}$-acetylmuramoylL-alanine amidase activity of the peptidoglycan recognition protein 2 (PGLYRP-2) destroys the Nod2-detected muramyl dipeptide structure. PGLYRP-2 green fluorescent protein (GFP) reporter and wild-type mice were studied by flow cytometry and quantitative RT-PCR to identify Pglyrp-2 expression in cells of the intestinal mucosa and reveal a potential regulatory function on epithelial peptidoglycan recognition. $\mathrm{CD}^{+} / \mathrm{CD} 11 \mathrm{c}^{+} \mathrm{T}$ lymphocytes revealed significant Pglyrp-2 expression, whereas epithelial cells and intestinal myeloid cells were negative. The mucosal Pglyrp-2-expressing lymphocyte population demonstrated a mixed T-cell receptor (TCR) $\alpha \beta$ or $\gamma \delta$ phenotype with predominant CD8 $\alpha$ and less so CD8 $\beta$ expression, as well as significant staining for the activation markers B220 and CD69, presenting a typical intraepithelial lymphocyte phenotype. Importantly, exposure of peptidoglycan to PGLYRP-2 significantly reduced Nod2/Rip2-mediated epithelial activation. Also, moderate but significant alterations of the intestinal microbiota composition were noted in Pglyrp-2-deficient animals. PGLYRP-2 might thus have a significant role in regulation of the enteric host-microbe homeostasis.

\section{INTRODUCTION}

The intestinal mucosa is colonized by a large variety and high number of commensal bacteria that significantly contribute to intestinal physiology. The epithelium is equipped to recognize the presence of microorganisms to control bacterial growth and maintain enteric homeostasis. Innate immune recognition is facilitated by several families of innate immune receptors that recognize conserved microbial structures and induce cellular activation. Expression of the cytosolic nucleotide-binding oligomerization domain (Nod) 2 receptor is found at the intestinal epithelium. Nod2 belongs to the family of Nod-like receptors and recognizes a muramyl dipeptide motif, structural subunit of the bacterial cell wall constituent peptidoglycan. ${ }^{1-3}$ Nod 2 has been associated with enteric antibacterial host defense and the regulation of the intestinal microbiota. ${ }^{4,5}$ Also, mutations within the Nod2 gene have been associated with the chronic intestinal inflammation in patients with Crohn's disease. ${ }^{6,7}$ Nod2 mutations were shown to suppress expression of the negative regulatory cytokine interleukin (IL)-10. ${ }^{8}$ Thus, in addition to innate immune receptor-stimulated host defense activation, negative regulatory mechanisms must also be present that prevent inappropriate immune stimulation by commensal bacteria leading to chronic inflammation and tissue destruction.

Peptidoglycan is constantly released by members of the enteric microbiota colonizing the intestinal mucosa. It comprises long alternating GlcNAc ( $N$-acetylglucosamine) and MurNAc ( $N$ acetylmuramic acid) glycan chains crosslinked by short peptide bridges bound to the lactic acid group on $\mathrm{N}$-acetylmuramic acid. The best-studied peptidoglycan-modifying enzyme is lysozyme secreted by macrophages and crypt Paneth cells. Lysozyme cleaves the $\beta 1-4$ glycosidic bond leaving the minimal Nod2-recognized structural motif muramyl (L-Ala D-Glu) dipeptide intact. In contrast, the $N$-acetylmuramoyl-L-alanine amidase peptidoglycan recognition protein 2 (PGLYRP-2, also

\footnotetext{
${ }^{1}$ Institute of Medical Microbiology and Hospital Epidemiology, Hannover Medical School, Hannover, Germany. ${ }^{2}$ Swedish Institute for Infectious Disease Control (SMI), Solna, Sweden. ${ }^{3}$ Division of Gastroenterology, Medical College of Wisconsin, Milwaukee, Wisconsin, USA. ${ }^{4}$ Microbiology and Tumor Biology Center (MTC), Karolinska Institutet, Stockholm, Sweden. ${ }^{5}$ Howard Hughes Medical Institute, Department of Medicine and Microbiology/Immunology, University of California San Francisco, San Francisco, California, USA. Correspondence: MW Hornef (hornef.mathias@mh-hannover.de) 
called PGRP-L) hydrolyses the lactyl bond between MurNAc and L-alanine and destroys the Nod2-recognized structure. ${ }^{9}$ PGLYRP-2 belongs to an evolutionary conserved family of peptidoglycan-binding proteins first identified in insects. ${ }^{10-12}$ Insect members of this protein family facilitate peptidoglycan recognition and host defense activation (hence the name) or show amidase activity and are involved in negative regulation of bacterial immune stimulation..$^{9,13-16}$ In mammals, four PGLYRPs have been described. ${ }^{11,17,18}$ They seem not to be involved in direct ligand recognition but exhibit enhanced expression upon immunostimulation. ${ }^{19}$ PGLYRP- 1 as well as PGLYRP-3 and - 4 are expressed in bone marrow, and esophagus, tonsils, and thymus, respectively, and exhibit significant bacterial killing activity. ${ }^{17,18,20}$ In contrast, the secreted protein PGLYRP-2 is mainly expressed in the liver and released into the bloodstream. It is devoid of antibacterial activity but similar to the Drosophila melanogaster PGRP-LB, and PGRP-SC1A/1B represents a catalytic amidase. ${ }^{13,14,16,18,21,22}$ Interestingly, the long-known peptidoglycan-degrading NAMLAA ( $\mathrm{N}$-acetylmuramoyl-L-alanine amidase activity) in human serum has only recently been attributed to the Pglyrp-2 gene. ${ }^{23,24}$ Although amidase pretreatment of peptidoglycan diminished the proinflammatory activity in vivo, Pglyrp-2-deficient mice did not exhibit an enhanced susceptibility to systemic bacterial infection. ${ }^{25,26}$ Intestinal mucosal PGLYRP-2 expression and the biologically relevant in vivo function are ill defined.

In this study, expression of PGLYRP- 2 by cells of the intestinal mucosa and the functional effect on epithelial Nod2-mediated peptidoglycan recognition was examined using mice with the green fluorescent protein (GFP) encoding gene replacing the start site in the first intron of Pglyrp-2 leaving all endogenous regulatory elements of Pglyrp-2 expression unaffected. The results for the first time demonstrate Pglyrp-2 expression by mucosal lymphocytes, characterize the lymphocyte population in great detail, and demonstrate a significant effect of PGLYRP-2 on intestinal epithelial peptidoglycan recognition. This work thus identifies a novel upstream regulatory mechanism of mucosal peptidoglycan recognition.

\section{RESULTS \\ PGLYRP-2 is expressed by epithelium-associated Iymphocytes}

Strong expression of mammalian PGLYRP-2 has been reported in the liver with secretion into the bloodstream. Indeed, flow cytometric analysis of liver cells isolated from mice expressing GFP under the control of the Pglyrp-2 regulatory elements revealed significant fluorescence (Supplementary Figure S1 online), and immunoblot analysis of serum from wild-type but not reporter mice detected PGLYRP-2 at the expected molecular weight (data not shown). As significant expression of Pglyrp-2 was also detected in total intestinal tissue, ${ }^{18}$ intestinal epithelial cells as well as associated lymphoid and myeloid cells were isolated from reporter animals and wild-type C57BL/6 mice and compared by flow cytometry. Highly enriched ( $>98 \%$ ) intestinal epithelial cells did not exhibit detectable fluorescence (Figure 1a). A significant population of $\mathrm{GFP}^{+}$cells was, however, detected in the preparation of epithelium-associated CD $45^{+}$cells, with $29.5 \%$ of $\mathrm{CD} 45^{+}$cells showing significant GFP expression (Figure 1b). Co-staining with markers for intestinal macrophages (F4/80) or polymorphonuclear cells (Gr-1) did not reveal expression by myeloid cells (Figure 1c) ${ }^{27-29}$ In contrast, 96.5\% $\mathrm{GFP}^{+}$cells exhibited staining for CD3 and $84.2 \%$ simultaneously expressed CD11c (Figure 1d), both characteristically expressed on intraepithelial lymphocytes. ${ }^{30,31}$ No significant CD11b or $\mathrm{CD} 19$ expression was noted on $\mathrm{GFP}^{+}$cells, indicating absence of reporter gene expression by intestinal mucosal dendritic cells or $\mathrm{B}$ lymphocytes, respectively (Figure 1d and e). GFP expression by intraepithelial lymphocyte cells was confirmed by immunohistological staining for GFP and CD3 in small intestinal tissue sections obtained from $g f p$ knock-in mice (Figure 1f). These results for the first time identify expression of the $N$-acetylmuramoylL-alanine amidase PGLYRP-2 by intraepithelial lymphocytes in close proximity to the microbial-exposed epithelium, suggesting a possible influence on mucosal peptidoglycan recognition.

\section{Detailed characterization of gfp-expressing epithelium- associated T Iymphocytes}

To investigate whether the $g f p$-expressing T lymphocyte population represented a defined lineage or whether Pglyrp-2 expression might display a common marker of mucosal T lymphocytes, the population of epithelium-associated $\mathrm{GFP}^{+}$cells was characterized for T-cell receptor (TCR $\alpha \beta$ and $\gamma \delta$ ) and co-receptor expression (CD4, CD $8 \alpha$, and CD $8 \beta$ ). Expression of the TCR $\alpha \beta$ and TCR $\gamma \delta$ was found in 50.0 and $45.9 \%$ of the $\mathrm{GFP}^{+}$population, respectively, (Figure $\mathbf{2} \mathbf{a}$ and $\mathbf{f}$ ). Of $\mathrm{GFP}^{+}$lymphocytes, $74.7 \%$ stained positive for the co-receptor CD $8 \alpha$ and $31.7 \%$ for $\mathrm{CD} 8 \beta$ (Figure $\mathbf{2 b}$ and $\mathbf{f}$ ). Of CD $8 \beta$-positive $\mathrm{GFP}^{+}$cells, $97.6 \%$ were also CD $8 \alpha$ positive, whereas $45.3 \%$ of $\mathrm{GFP}^{+}$lymphocytes expressed only CD8 $\alpha$. Of GFP ${ }^{+}$lymphocytes, $16.7 \%$ showed CD4 expression and only a small fraction of $1.1 \%$ CD $4 / C D 8 \alpha$ double-positive and $0.2 \% \mathrm{CD} 4 / \mathrm{CD} 8 \beta$ double-positive cells were noted among $\mathrm{GFP}^{+}$lymphocytes. Of CD4-positive $\mathrm{GFP}^{+}$lymphocytes, $85.8 \%$ stained positive for the TCR $\alpha \beta$ receptor. The percentage of $\mathrm{GFP}^{+}$cells among $\mathrm{CD} 3^{+}, \mathrm{TCR} \alpha \beta^{+}, \mathrm{TCR} \gamma \delta^{+}$, $\mathrm{CD} 8 \alpha^{+}, \mathrm{CD} 8 \beta^{+}$, and $\mathrm{CD} 4^{+}$intraepithelial lymphocytes is shown in Supplementary Table S1 online.

CD69, an early surface protein characteristic for activated T lymphocytes, ${ }^{32}$ was present on $93.1 \% \mathrm{GFP}^{+}$lymphocytes (Figure $2 \mathbf{c}$ and $\mathbf{f}$ ). Respectively, 79.4 and $93.6 \%$ of GFP ${ }^{+}$TCR $\alpha \beta$ and TCR $\gamma \delta$-positive lymphocytes also stained for CD69. Also, 98.0\% of CD8 $\alpha$-positive GFP ${ }^{+}$lymphocytes expressed CD69. In addition, staining for the marker of regulatory T cells Foxp3 (forkhead box P3) was performed on $\mathrm{GFP}^{+}$cells following sorting by flow cytometry (Figure 2d ). Regulatory $\mathrm{T}$ cells have an important role in gut homeostasis. ${ }^{33}$ Yet, $<1.0 \%$ Foxp3-positive cells were detected among sorted $\mathrm{GFP}^{+}$intraepithelial lymphocytes in accordance with the low number of Foxp3-positive cells among intraepithelial lymphocytes indicated in the recent literature. ${ }^{34}$ A significant influence of PGLYRP-2 expression on the development and tissue distribution of Foxp ${ }^{+}$regulatory $\mathrm{T}$ lymphocytes was excluded by a comparative analysis of the number of Foxp $3^{+}$cells in spleen and intestinal lamina 

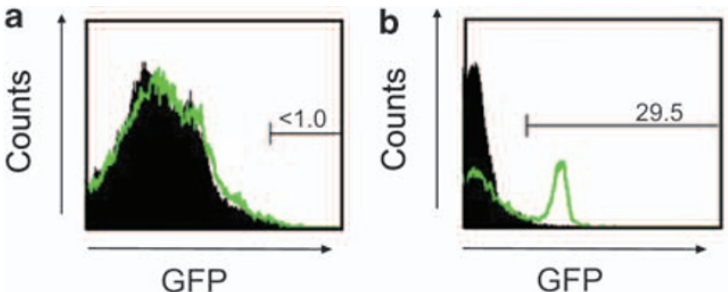

GFP
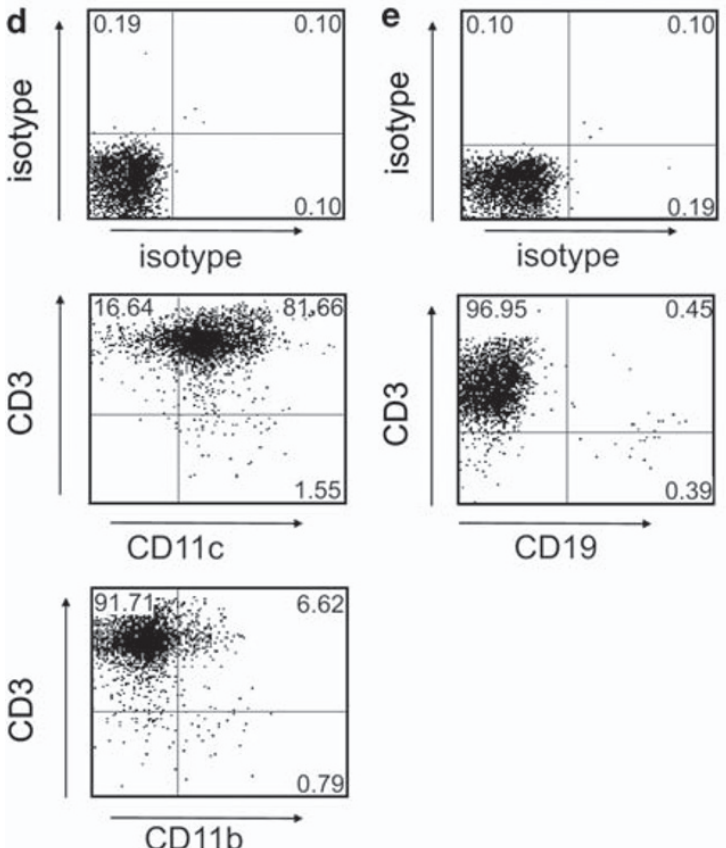

CD19

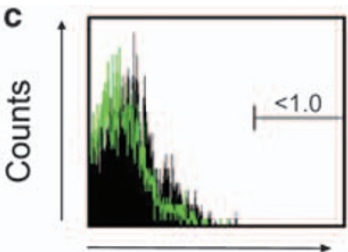

$\mathrm{F} 4 / 80$

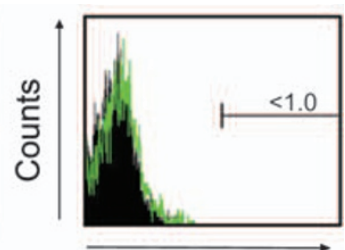

GR1 f
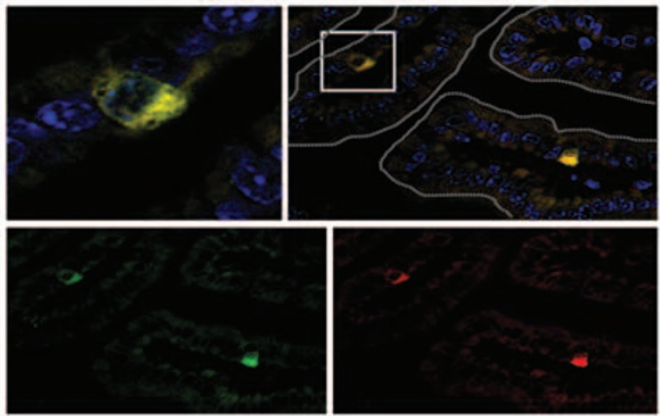

Figure 1 Reporter gene expression in mice carrying the green fluorescent protein (GFP) under the control of the Pglyrp-2 promoter. (a, b) GFP expression in intestinal mucosal cells of C57BL/6 wild-type vs. gfp knock-in reporter mice. (a) Primary intestinal epithelial cells were prepared from wild-type and gfp knock-in C57BL/6 mice and analyzed by flow cytometry for GFP expression (FL-1). The histogram shows fluorescence of the total epithelial cell preparation. (b) Fluorescence intensity of CD45+ epithelium-associated cells from C57BL/6 wild-type and reporter mice as determined by flow cytometry. (c) Histograms demonstrating expression of the myeloid surface markers F4/80 and Gr-1 on gated GFP ${ }^{+}$primary epithelium-associated cells. (d, e) Simultaneous CD3/CD11c and CD3/CD11b staining (d) as well as CD3/CD19 staining (e) of GFP+ epithelium-associated cells shown by dot blot. (f) Immunostaining for CD3 (red) and GFP (green) to visualize GFP-expressing intraepithelial lymphocytes in small intestinal tissue of gfp knockin reporter mice. The epithelial surface is highlighted by the grey dotted line; the white square indicates the insert shown as magnified image in the left upper panel. Original magnification $\times 630$. Each flow cytometric analysis was performed on cells obtained individually from 3 to 4 mice. A representative analysis is presented and the statistical analysis of all performed experiments is indicated in the text and Figure $\mathbf{2 f}$.

propria tissue from wild-type and Pglyrp-2-deficient mice (Supplementary Figure S2 online).

Mucosal GFP ${ }^{+}$T lymphocytes were tested for expression of the additional cell surface markers CD137, CD27, and B220 (Figure $2 \mathbf{e}$ and $\mathbf{f}$ ). Only $2.4 \%$ of $\mathrm{GFP}^{+} \mathrm{CD}^{+} \mathrm{T}$ lymphocytes expressed CD137, a co-stimulator of proliferation ${ }^{35}$ (Figure 2d). Of the $\mathrm{GFP}^{+} \mathrm{CD}^{+}$population, $26.3 \%$ was also positive for the surface marker CD27 in accordance with the recent report on CD27 expression by gut-resident $\gamma \delta$ T lymphocytes. ${ }^{36}$ Finally, $46.0 \%$ of the reporter gene expressing T lymphocytes showed B220 surface expression. The CD45 isoform B220, normally used to identify B cells, also represents a marker for activated $\mathrm{T}$ lymphocytes ${ }^{37-40}$ (Figure 2e). The expression of the various marker proteins on small intestinal intraepithelial $\mathrm{GFP}^{+}$lymphocytes is summarized in Figure 2f.

It is noteworthy that Pglyrp-2 expression is not restricted to small intestinal tissue. $\mathrm{GFP}^{+}$cells were also noted in spleen tissue and among intraepithelial lymphocytes in colon tissue isolated from $g f p$ transgene animals (Supplementary Figure $\mathbf{S 3}$ online). When compared with the small intestinal cells, GFP ${ }^{+}$ intraepithelial lymphocytes in colon tissue expressed more TCR $\alpha \beta(62.9 \%)$ and less TCR $\gamma \delta$ (20.1\%) (Supplementary Figure S3A online). The distribution of co-receptor expression was similar with the highest percentage of cells being positive for $\mathrm{CD} 8 \alpha$ (59.0\%), less so for $\mathrm{CD} 8 \beta$ (27.4\%), and only few cells expressing CD4 (3.0\%) (Supplementary Figure S3A online). In spleen tissue, $\mathrm{GFP}^{+}$cells expressed TCR $\alpha \beta$ (60.1\%) but not TCR $\gamma \delta(1.6 \%)$, as expected (Supplementary Figure S3B online). Also, spleen cells exhibited a balanced CD $8 \alpha$ (32.6\%), CD8 $\beta$ (29.9\%), and CD4 (31.6\%) distribution (Supplementary Figure S3B online). Together, Pglyrp-2 expression seems not to be attributed to a specific lymphocyte lineage but rather to represent a general feature of intraepithelial T lymphocytes in small intestine and colon as well as of spleen T cells. Pglyrp-2 is expressed by small intestinal T lymphocytes exhibiting a typical activated $\left(\mathrm{B}_{2} 20^{+}\right.$and 

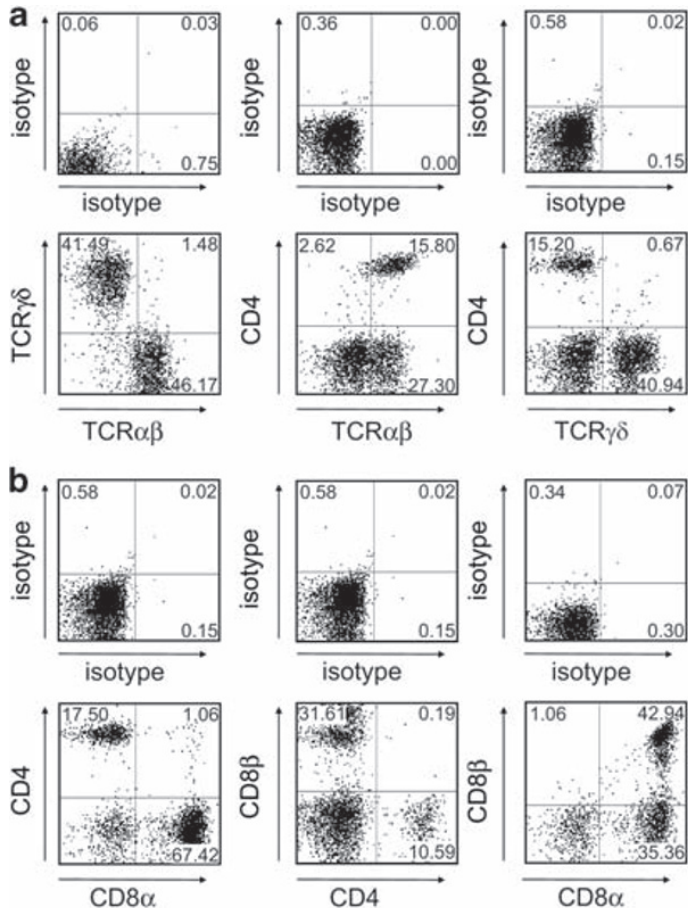
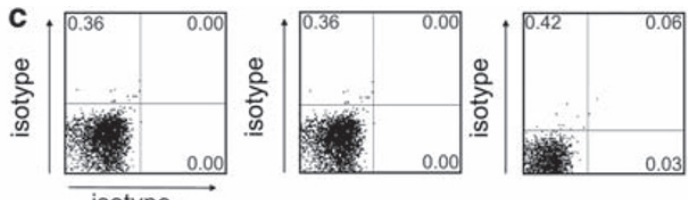

\section{d}
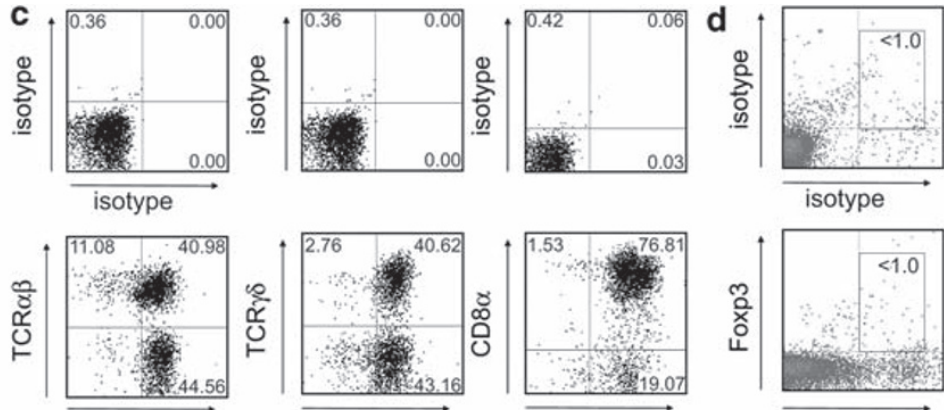

CD69

CD69
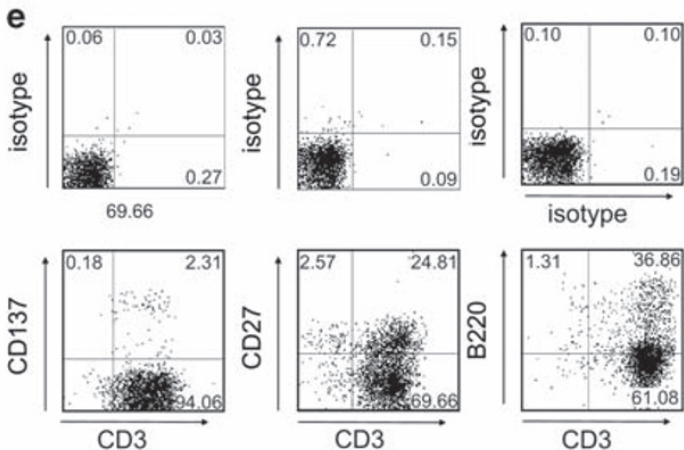

isotype

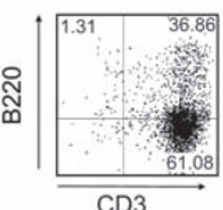

CD3

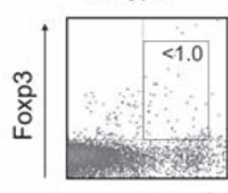

CD4

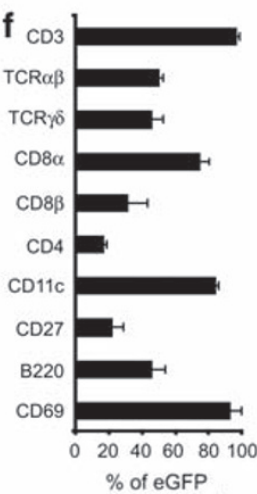

Figure 2 Detailed characterization of the reporter gene expressing intestinal T lymphocyte population. Green fluorescent protein-positive (GFP $\left.{ }^{+}\right)$cells from primary isolated epithelium-associated cells were analyzed for the expression of various surface marker proteins by flow cytometry. The numbers in the dot blot images indicate the percentage of single or double positive cells for the indicated marker protein among $\mathrm{GFP}^{+}$cells of the presented representative experiment. (a) T-cell receptor (TCR) $\alpha \beta, \mathrm{TCR} \gamma \delta$, and CD4 expression and (b) co-receptor CD4, CD8 $\alpha$, and CD8 $\beta$ expression by GFP ${ }^{+}$ mucosal cells isolated from transgene animals. (c) CD69 expression in combination with TCR $\alpha \beta, \mathrm{TCR} \gamma \delta$, and CD $8 \alpha$. (d) GFP ${ }^{+}$cells were isolated from total mucosal cells by flow cytometric sorting and subsequently stained for intracellular detection of Foxp3 (forkhead box P3) by flow cytometry. Spleen cells stained for Foxp3 shown in Supplementary Figure S2 online serve as positive control for the Foxp3 staining. (e) CD137, CD27, and B220 expression in combination with CD3 by GFP ${ }^{+}$cells. Each flow cytometric analysis was performed on cells obtained individually from 3 to 4 mice. A representative analysis is presented and the statistical analysis (mean \pm s.d.) of all performed results is summarized in a column diagram (f).

$\left.\mathrm{CD} 69^{+}\right)$nonproliferative $\left(\mathrm{CD} 137^{-}\right)$intestinal intraepithelial lymphocyte phenotype.

\section{Confirmation of the lymphocyte phenotype by flow cytometric sorting and RT-PCR analysis}

Expression of Pglyrp-2 by intraepithelial T lymphocytes was subsequently confirmed in C57BL/6 wild-type mice by quantitative RT-PCR. In accordance with previous results, strong Pglyrp-2 expression was detected in liver tissue (Figure 3a). ${ }^{18}$ Also, isolated epithelium-associated cells but not intestinal epithelial cells revealed significant Pglyrp-2 mRNA expression. To further verify the cellular expression pattern in wild-type mucosal cells, isolated epithelium-associated cells were sorted by flow cytometry for expression of CD3 alone or in combination with TCR $\alpha \beta, \mathrm{TCR} \gamma \delta, \mathrm{CD} 4, \mathrm{CD} 8 \alpha$, $\mathrm{CD} 8 \beta, \mathrm{B} 220$, or CD27, respectively (Figure $3 \mathbf{b}$ ). High expression of Pglyrp-2 was detected in $\mathrm{CD} 3^{+}$T lymphocytes with similar expression levels in both $\mathrm{CD}^{+} / \mathrm{TCR} \alpha \beta^{+}$and $\mathrm{CD} 3^{+} /$ $\mathrm{TCR} \gamma \delta^{+}$cells. Whereas $\mathrm{CD}^{+} / \mathrm{CD} 8^{+}$lymphocytes exhibited high gene expression, lower values were obtained for $\mathrm{CD} 3{ }^{+} / \mathrm{CD} 4^{+}$lymphocytes.

The finding that expression was detected in $\mathrm{CD} 9^{+}$activated mucosal T lymphocytes prompted us to investigate the influence of exogenous and endogenous immune stimuli on Pglyrp-2 expression by intraepithelial lymphocytes. No significant difference in the Pglyrp-2 mRNA expression level was detected in intraepithelial lymphocytes prepared from Nod2-deficient when compared with wild-type mice (Figure 4a). In addition, no influence of exogenous microbial stimuli such as lipopolysaccharide (LPS) and peptidoglycan or endogenous immunoregulatory mediators such as IL-15 and IL-22 on Pglyrp-2 expression was observed (Figure 4b). Even in the presence of anti-CD3/antiCD28 antibodies, stimulation with LPS, peptidoglycan, IL-15, or IL-22 did not lead to significant changes in Pglyrp-2 mRNA levels (Figure 4c). These results identify strong constitutive, T lymphocyte lineage-independent expression of Pglyrp-2 in intestinal intraepithelial lymphocytes that might represent a new mechanism to limit the concentration of stimulatory mucosal peptidoglycan.

\section{PGLYRP-2 influences intestinal epithelial peptidoglycan recognition}

The $N$-acetylmuramoyl-L-alanine amidase activity of PGLYRP2 destroys the Nod2-recognized structural muramyl dipeptide motif of peptidoglycan. Expression of the peptidoglycan receptor Nod2 has been shown to have an important role in the maintenance of the enteric homeostasis. ${ }^{4,5}$ Despite expression of Nod2 in isolated intraepithelial T lymphocytes (Figure 5a), 

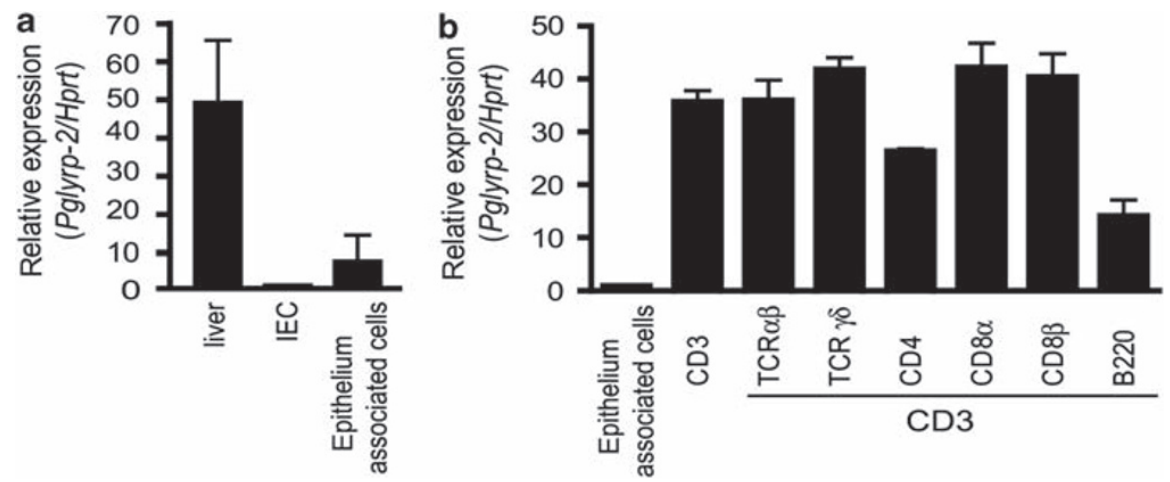

Figure 3 Confirmation of Pglyrp-2 expression in primary intestinal mucosal C57BL/6 wild-type cells by quantitative RT-PCR. (a) RNA was isolated from liver tissue, isolated primary intestinal epithelial cells, and intestinal epithelium-associated cells, and quantitative RT-PCR for Pglyrp-2 in relation to the Hprt housekeeping gene was performed. (b) Primary intestinal epithelium-associated cells were stained for expression of CD3 alone or CD3 in combination with T-cell receptor (TCR) $\alpha \beta, \mathrm{TCR} \gamma \delta, \mathrm{CD} 4, \mathrm{CD} 8 \alpha, \mathrm{CD} 8 \beta, \mathrm{B} 220$, or CD27, respectively, and sorted by flow cytometry. Pglyrp-2 in relation to the Hprt expression was analyzed by quantitative RT-PCR and is shown as fold change. For each parameter, cells were prepared from 3 to 4 mice, sorted, and analyzed individually for Pglyrp-2 mRNA expression. Mean \pm s.d. is shown.
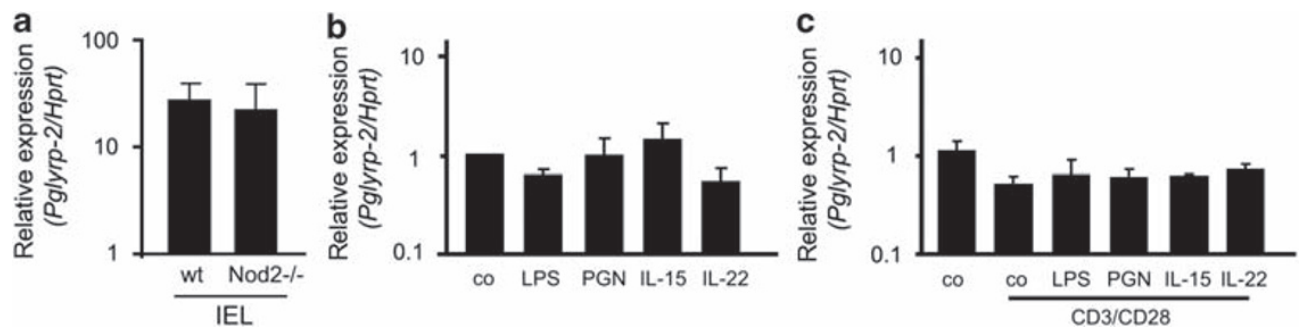

Figure 4 Regulation of Pglyrp-2 expression in mucosal lymphocytes by endogenous and exogenous stimuli. (a) Expression of peptidoglycan recognition protein 2 (Pglyrp-2) in intraepithelial lymphocytes (IELs) obtained from wild-type $(n=5)$ and nucleotide oligomerization domain 2 (Nod2)deficient mice $(n=5)$. Quantitative RT-PCR for Pglyrp-2 was performed in total mRNA prepared from primary IELs and normalized to the Hprt housekeeping gene. The values indicate Pglyrp-2 mRNA levels relative to Pglyrp-2 expression in IECs. (b, c) Influence of endogenous and exogenous stimuli on Pglyrp-2 expression in primary IELs. Quantitative RT-PCR analysis for Pglyrp-2 mRNA expression normalized to the Hprt housekeeping gene was performed using total mRNA prepared from primary IELs after incubation for $18 \mathrm{~h}$ at $37^{\circ} \mathrm{C}$ in the presence of lipopolysaccharide (LPS, $10 \mathrm{ng} \mathrm{ml}^{-1}$ ), peptidoglycan (PG, $10 \mu \mathrm{g} \mathrm{ml}^{-1}$ ), interleukin (IL)-15, and IL-22 (each $50 \mathrm{ng} \mathrm{ml}^{-1}$ ) in the (b) absence or (c) presence of anti-CD3 $\left(1 \mu \mathrm{g} \mathrm{ml} \mathrm{C}^{-1}\right.$ ) and anti-CD28 $\left(2 \mu \mathrm{g} \mathrm{ml}^{-1}\right)$ and is presented as relative change. All experiments were repeated at least twice and a representative result is presented.

no interferon (IFN)- $\gamma$ production in response to peptidoglycan stimulation was detected (Figure 5b). As PGLYRP-2 represents a secreted protein and intraepithelial lymphocytes reside in close proximity to the enteric mucosal surface, we next studied the response of intestinal epithelial cells to peptidoglycan and the influence of PGLYRP-2 on epithelial Nod2 recognition. As expected, Nod2 expression was detected in primary isolated intestinal epithelial cells and intestinal epithelial $\mathrm{m}-\mathrm{IC}_{\mathrm{cl} 2}$ cells (Figure 5a, and data not shown) with upregulation of Nod2 expression in response to microbial stimulation (Figure 5c). In contrast to intraepithelial lymphocytes, $\mathrm{m}-\mathrm{IC}_{\mathrm{cl} 2}$ cells responded to peptidoglycan with the secretion of the proinflammatory chemokine macrophage inflammatory protein-2 in a time- and dose-dependent manner (Figure 5d and Supplementary Figure 4A and B online). Small interfering RNA (siRNA)-mediated gene downregulation confirmed that the epithelial peptidoglycan response was dependent on expression of the Nod2 receptor $(P<0.05)$ and the downstream signaling molecule Rip2 $(P<0.05)$, but independent of the innate immune receptors Nod 1 and Toll-like receptor (TLR)2 (Figure 5d). The residual response to peptidoglycan after Nod2 or Rip2 siRNA treatment was most probably due to a limited transfection efficiency and gene downregulation in intestinal epithelial $\mathrm{m}-\mathrm{IC}_{\mathrm{c} 2}$ cells by this technique. Importantly, pretreatment with recombinant PGLYRP-2 significantly reduced the immunostimulatory effect of peptidoglycan (Figure 5e) but not LPS (Figure 5f). The potency of the effect was similar to the reduced chemokine secretion observed after siRNA-mediated Nod2 downregulation. These results are in accordance with a catalytic degradation of the stimulatory activity of peptidoglycan and indicate that the $\mathrm{N}$-acetylmuramoyl-L-amidase activity of PGLYRP-2 might significantly impair the stimulatory potential of peptidoglycan.

To examine a possible impact of Pglyrp-2-induced alterations of the Nod2-mediated peptidoglycan recognition on the enteric host-microbial interaction, the bacterial microbiota composition in the proximal and distal small intestine as well as large intestine was comparatively analyzed in wild-type, Pglyrp-2-, and Nod2deficient animals by quantitative PCR using bacterial groupspecific $16 \mathrm{~S}$ rDNA primers. ${ }^{41}$ Whereas the bacterial composition in the large intestine of wild-type, Pglyrp-2-, and Nod2-deficient animals was very similar, differences in the gene copy number of the Bacteroides group $(P=0.017)$ and the Mouse Intestinal Bacteroides group $(P=0.004)$ were noted in the distal small 


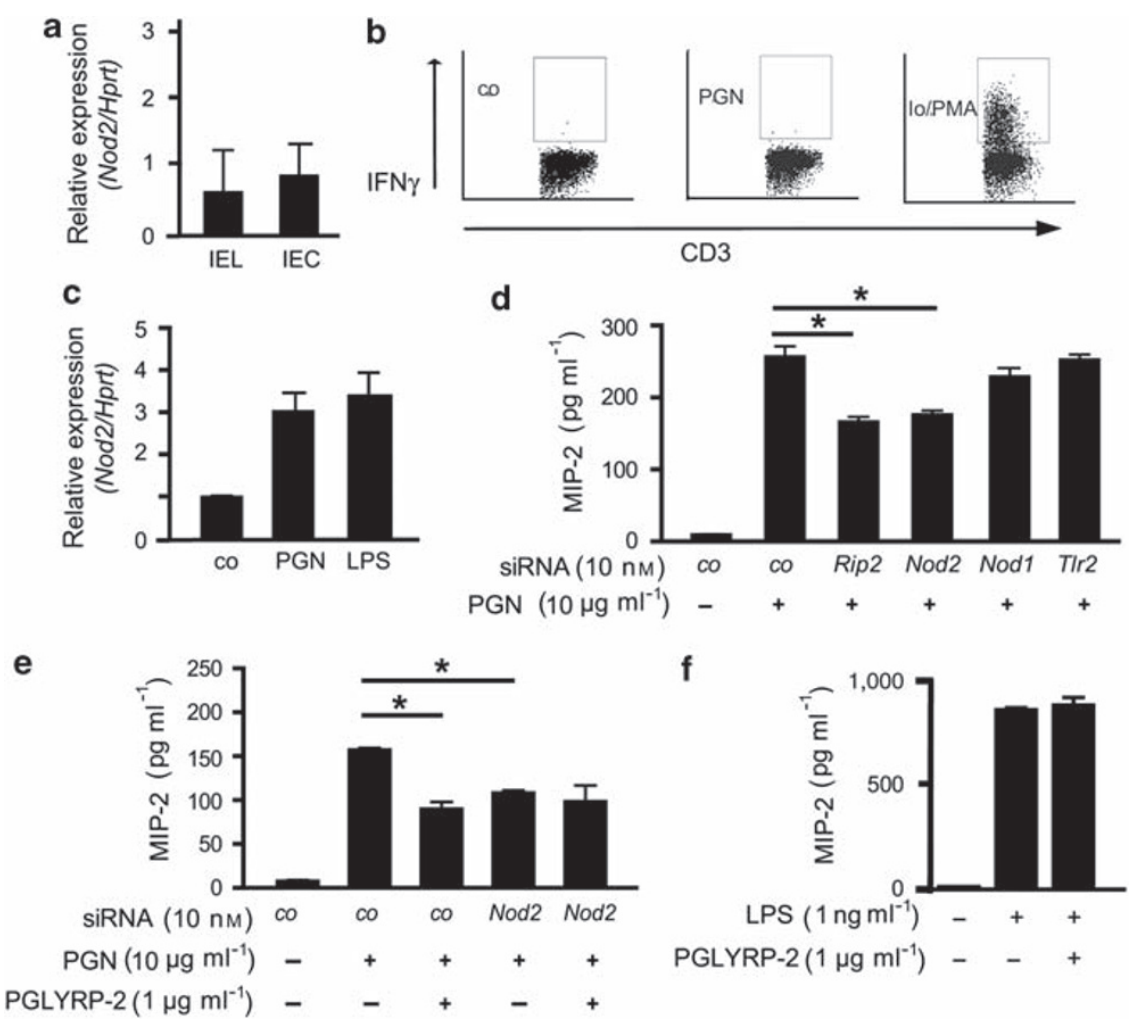

Figure 5 Analysis of the effect of peptidoglycan recognition protein 2 (PGLYRP-2) on intestinal epithelial nucleotide oligomerization domain (Nod2)mediated peptidoglycan (PG) recognition. (a) Quantitative RT-PCR for Nod2 normalized to the Hprt expression in isolated intraepithelial lymphocytes (IELs) and primary intestinal epithelial cells (IECs) is shown as fold change. (b) Flow cytometric analysis of intracellular interferon (IFN)- $\gamma$ staining in gated $C D 3^{+}$primary IELs after incubation for $4 \mathrm{~h}$ in the presence of $10 \mu \mathrm{g} \mathrm{ml}^{-1} \mathrm{PG}$. Brefeldin A was added after $2 \mathrm{~h}$ of incubation. Ionomycin/phorbol myristate acetate (Io/PMA) served as positive control. (c) Quantitative RT-PCR for Nod2 expression in intestinal epithelial m-IC $\mathrm{cl}_{2}$ cells left untreated or incubated in the presence of lipopolysaccharide (LPS, $\left.10 \mathrm{ng} \mathrm{ml}^{-1}\right)$ or PG $\left(10 \mu \mathrm{g} \mathrm{ml}^{-1}\right)$ for $6 \mathrm{~h}$ normalized to Hprt is shown as fold change. (d) Secretion of the chemokine macrophage inflammatory protein 2 (MIP-2) by intestinal epithelial mIC ${ }_{\mathrm{cl} 2}$ cells transfected with control (co), Rip2-, Nod2- , Nod1-, and TIr2-specific small interfering RNA (siRNA) before stimulation with PG $\left(10 \mu \mathrm{g} \mathrm{ml}^{-1}\right)$ for $6 \mathrm{~h}$. ${ }^{\star} P<0.05$. (e, f) MIP-2 secretion by control or Nod2specific siRNA-transfected $\mathrm{mIC}_{\mathrm{cl} 2}$ cells after exposure to (e) untreated PG or PG pretreated with recombinant PGLYRP-2 and (f) untreated LPS or LPS pretreated with recombinant PGLYRP-2. ${ }^{\star} P<0.05$. All experiments were repeated at least twice and a representative result is presented.

intestine and of the Lactobacillus/Enterococcus group $(P=0.019)$ and the segmented filamentous bacteria group $(P=0.006)$ were noted between wild-type and Pglyrp-2-deficient animals in the proximal small intestine. The gene copy number of segmented filamentous bacteria in the proximal small intestine also exhibited a significant difference between wild-type and Nod2-deficient animals $(P<0.001$; Figure 6 and Supplementary Table S2 online). These results are in accordance with an upstream regulatory function of PGLYRP-2 on Nod2-mediated innate immune effector activities that influence the bacterial composition at the small intestinal mucosa.

\section{DISCUSSION}

In D. melanogaster, the family of peptidoglycan recognition proteins (PGRPs) have an essential role in immunity. PGRPs directly bind to the bacterial cell wall component peptidoglycan and initiate the signaling cascade leading to immune stimulation and antibacterial and antifungal host defense. In addition, certain isoforms such as PGRP-LB possess amidase activity and inactivate the recognized bacterial ligand. As intestinal PGRPLB expression is upregulated by immune stimulation, its luminal secretion represents an upstream negative regulatory mechanism to restrict the presence of active ligand and terminate and downregulate host defense activation..$^{16}$ Although the important role of Nod2 for intestinal homeostasis has been established, the role of catalytic amidase activity within the mammalian intestine has not been examined. Yet, this appears to be of particular interest as a similar catalytic negative regulatory mechanism upstream of bacterial LPS recognition has recently been described in the fish and mouse intestine. ${ }^{42,43}$ Intestinal alkaline phosphatase removes one phosphate group from LipidA, the essential constituent and structural motif recognized by the LPS immune receptor TLR4. Intestinal alkaline phosphatase is secreted into the intestinal lumen, and by dephosphorylation of LPS restricts innate immune activation and the recruitment of professional immune cells to the enteric mucosa. ${ }^{43}$

In this study, we analyzed Pglyrp-2 expression by cells of the murine intestinal mucosa and studied the effect on intestinal epithelial peptidoglycan recognition. To our surprise, we observed significant Pglyrp-2 expression by intraepithelial T lymphocytes in addition to the previously observed production of PGLYRP-2 in liver tissue. The characterization of PGLYRP2-positive lymphocytes in the small intestinal mucosa revealed expression by $\mathrm{CD} 3^{+}, \mathrm{TCR} \alpha \beta^{+}$or $\mathrm{TCR} \gamma \delta^{+}$, mainly $\mathrm{CD} 8^{+}$, partly 


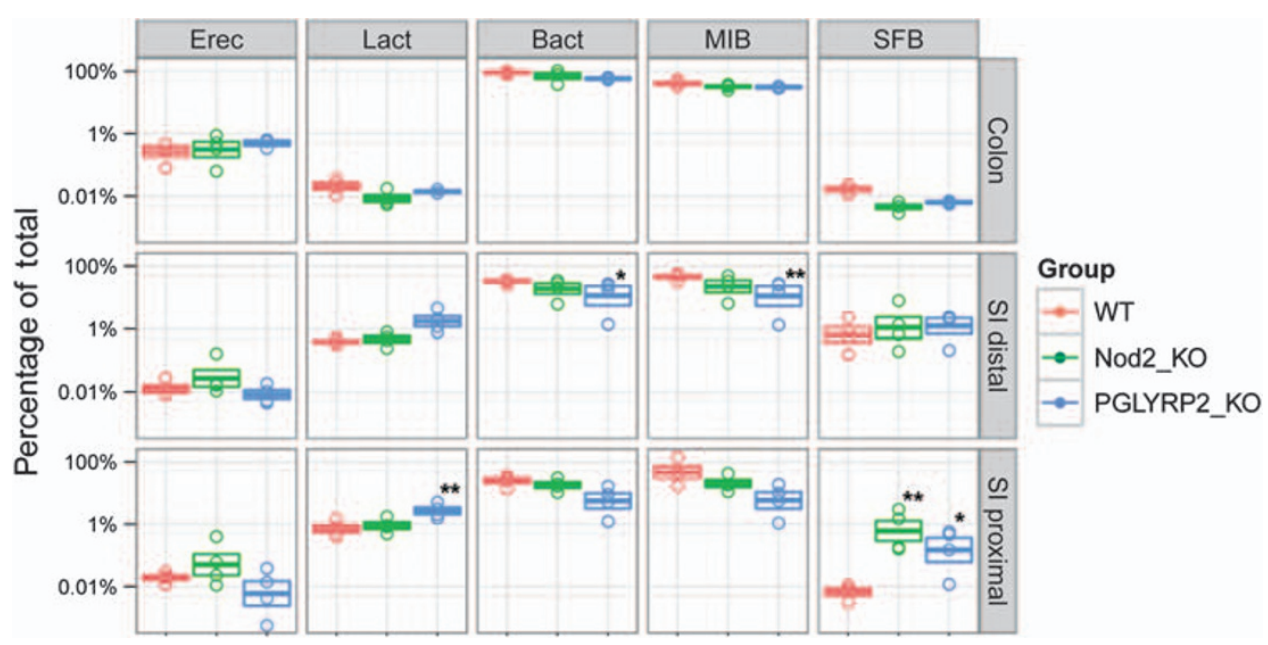

Figure 6 Comparative analysis of the bacterial microbiota in proximal and distal small intestine and colon of wild-type, Nod2-, and Pglyrp-2 deficient mice. Quantitative 16S rDNA PCR of the indicated bacterial groups in total proximal or distal small intestinal or colon tissue homogenate. Tissue from four mice was analyzed in each group. The numbers relative to the total bacteria present are indicated in percentages. The points are the actual observations, with the boxes showing the mean with one-standard error intervals. The following bacterial groups are shown: Erec, Eubacterium rectale/Clostridium coccoides group; Lact, Lactobacillus/Enterococcus group; Bact, Bacteroides; MIB, mouse intestinal Bacteroides; SFB, segmented filamentous bacteria. ${ }^{*} P<0.05 ;{ }^{* *} P<0.01$.

$\mathrm{CD} 9^{+}$and $\mathrm{B} 220^{+}$activated, $\mathrm{CD} 137^{-}$nonproliferative intestinal intraepithelial T lymphocytes. Thus, PGLYRP-2 expression in the small intestine is found in typical gut-associated activated effector or memory $\mathrm{T}$ lymphocytes that are situated in close proximity to the epithelium. PGLYRP-2 production, however, was also noted in colon intraepithelial lymphocytes and spleen cells and appeared not to be restricted to a distinct T-cell lineage but rather to be linked to the Tlymphocyte composition in a given anatomical environment. ${ }^{44}$

In contrast to the situation in D. melanogaster, amidase production was not significantly altered by microbial immunostimulation or endogeneous homeostatic cytokines IL-15 or IL-22. Also, no significant difference of Pglyrp-2 mRNA was noted between wild-type and Nod2-deficient animals, despite the fact that alterations in the lymphoid development have been described. ${ }^{45}$ Mammalian PGLYRP-2 expression might therefore represent a constitutive part of the immunoregulatory network involved to prevent inappropriate stimulation by the microbiota of the intestine. Yet, we cannot exclude a regulatory effect of unidentified exogenous or endogenous mediators.

We did not detect IFN- $\gamma$ production by $\mathrm{T}$ lymphocytes in response to stimulation with the Nod2 ligand peptidoglycan. Even though these results do not exclude a functional effect of peptidoglycan on intraepithelial lymphocytes, they suggest that other cell types might represent the primary target for luminal peptidoglycan. Indeed, intestinal epithelial cells significantly responded to peptidoglycan in a time- and dose-dependent manner, and a significant influence of PGLYRP-2 on epithelial peptidoglycan recognition was observed. Recent reports about an important role of Nod2 for gut homeostasis and the differences in the small intestinal microbiota composition between wild-type and Pglyrp-2-deficient mice identified in this study suggest that PGLYRP-2 secretion might have a direct or indirect effect on the mucosal mechanisms that regulate the enteric host-microbial homeostasis. ${ }^{5}$ For example, the observed proinflammatory response of the intestinal mucosa of germ-free mice in response to bacterial colonization might-at least in part-be attributed to PGLYRP-2 deficiency, as germ-free housed mice have a severely underdeveloped intestinal lymphogenesis. ${ }^{45}$ Also, a significant increase in the number of mucosal effector $\mathrm{T}$ lymphocytes in addition to regulatory $\mathrm{T}$ cells has been described under inflammatory conditions such as in patients with inflammatory bowel disease who also show significant alteration in their enteric microbiota composition. ${ }^{46}$ Yet, it is clear that the mechanisms that influence the enteric microbiota are incompletely understood and the identification of the underlying regulatory circuits certainly requires further attention. For example, the biological in vivo effect of $\beta 1-4$ glycosidic bond cleaving lysozyme or amidase activity on peptidoglycan degradation, fragment internalization, and immune stimulation at the intestinal mucosa is undefined.

The recent description of a systemic effect of gut-derived peptidoglycan, for example, on bone marrow cells possibly also extends the functional relevance of our findings to nonenteric anatomical sites. ${ }^{47}$ PGLYRP-2-deficient mice, however, do not exhibit any spontaneous phenotype, similar to what has been described for Nod2-deficient animals. ${ }^{4,26}$ Interestingly, a recent study demonstrated a protective role of PGLYRP-2 in a dextran sulfate sodium-induced colitis model. ${ }^{48}$ In this study, PGLYRP-2, similar to other PGLYRP family members, was shown to prevent IFN- $\gamma$-mediated natural killer cell recruitment and mucosal tissue damage following oral dextran sulfate sodium administration. In accordance with our results, moderate changes in the intestinal microbiota composition were noted and linked to the enhanced susceptibility to mucosal inflammation. For example, enhanced numbers of the group of segmented filamentous bacteria have previously been associated with a more proinflammatory reaction in an 
experimental autoimmune encephalomyelitis and T-cell transfer colitis model. ${ }^{49,50}$ Interestingly, whereas Pglyrp-2 expression protected from dextran sulfate sodium-induced colitis, it promoted inflammation in a peptidoglycan-induced arthritis model ${ }^{48,51}$ Both Nod2 and PGLYRP-2 seem to be involved in the severity of inflammatory arthritis after local tissue instillation of peptidoglycan. ${ }^{51-53}$ Parenteral injection of PGLYRP-2, however, had no effect on the disease development, and the source of PGLYRP-2 involved in the pathogenesis was not examined. It is noteworthy that these studies were performed using mice on the Balb/c genetic background in contrast to our work.

In conclusion, this is the first report characterizing intestinal mucosal Pglyrp-2 expression in detail and demonstrating its inhibitory effect on intestinal epithelial peptidoglycan recognition. Pglyrp-2 expression is detected in intraepithelial $\mathrm{CD} 8^{+} \mathrm{TCR} \alpha \beta^{+}$or TCR $\gamma \delta^{+}$T lymphocytes. As PGLYRP-2 irreversible destroys the muramyldipeptide motif detected by Nod2, mucosal expression might significantly diminish innate immune recognition of bacterial peptidoglycan, leading to an altered host-microbial balance. Our results thus identify a novel upstream regulatory mechanism of intestinal Nod2 recognition in accordance with recent reports on the close interaction between intestinal epithelial cells and intraepithelial lymphoid cells in the context of microbial colonization. ${ }^{54,55}$ This illustrates the cooperative function of cells of the adaptive immune system on epithelial innate immune recognition to restrict inappropriate host defense activation at the intestinal epithelial surface.

\section{METHODS}

Antibodies and reagents. Fluorophore-conjugated antibodies directed against CD3, CD $8 \alpha, \mathrm{CD} 8 \beta, \mathrm{CD} 4, \mathrm{CD} 27, \mathrm{~B} 220$, TCR $\alpha \beta$, TCR $\gamma \delta$, FoxP3, F4/80, CD19, CD11b, and CD11c were purchased from NatuTec GmbH/eBioscience (Frankfurt, Germany) and BIOZOL Diagnostica Vertrieb GmbH (München, Germany). Unconjugated CD3 and CD28 were obtained from eBioscience (Frankfurt, Germany). The Cy5-conjugated anti-CD3 antibody was obtained from Abcam (Cambridge, UK). Biotinylated antibodies directed against CD11c, F4/80 (clone BM8), and GR-1 were from BD Pharmingen (Heidelberg, Germany), and CD69 from NatuTec GmbH/eBioscience and detected using Cy5conjugated streptavidin from Dianova (Hamburg, Germany). For blocking Mouse BD Fc Block was used from BD Pharmingen. All siRNA probes employed in this study (Nod1, Nod2, Tlr2, Rip2, and control siRNA) were purchased from Qiagen (Hilden, Germany). For siRNA transfection INTERFERin (Polyplus Transfection, New York, NY) was used according to the manufacturer's instructions. The efficacy of Nod1, Nod2, Tlr2, Rip2, and siRNA treatment is illustrated in ref. 56. Cell culture reagents were from Invitrogen (Karlsruhe, Germany). Recombinant cytokines (IL-22 and IL-15) were purchased from PeproTech (Hamburg, Germany). Total RNA extraction was performed using Trizol from Invitrogen; RNA from sorted cells was isolated using the RNA micro kit from Qiagen. Complementary DNA was synthesized using the Transcriptor First Strand cDNA Synthesis Kit from Roche (Penzberg, Germany) and RT-PCR was performed using the Taqman Gene Expression assay for mouse Pglyrp-2 (Mm00480457) Nod2 (Mm00467543), and Hprt-1 (Mm00446968) from Applied Biosystems (Darmstadt, Germany) according to the manufacturer's recommendations and relative expression (target gene/Hprt) is shown in fold change.
Percoll was purchased from GE Healthcare (Uppsala, Sweden) and collagenase A from Roche. Recombinant PGLYRP-2 was prepared as described previously. ${ }^{21}$ Briefly, His-tagged PGLYRP-2 was expressed in S2 Schneider cells and purified over a Ni-sepharose column. Before cell stimulation, peptidoglycan was pretreated for $30 \mathrm{~min}$ at $37^{\circ} \mathrm{C}$ with recombinant PGLYRP-2. All other reagents were obtained from Sigma (Taufkirchen, Germany) if not stated otherwise.

Animals. All animals were housed under specific pathogen-free conditions in accordance with the local regulations. The PGLYRP-2 GFP reporter mice were created by replacing the start site in the first intron of the Pglyrp-2 gene with eGFP leaving all endogenous regulatory elements of Pglyrp-2 expression unaffected. The targeted embryonic 129/SVJaederived stem cells were injected into C57BL/6 mice and after loss of the selection cassette mice were backcrossed to $\mathrm{C} 57 \mathrm{BL} / 6$ for ten generations and made homozygous to create null animals as previously reported. ${ }^{26}$ No PGLYRP-2 protein expression was detected in homozygous $g f p$ reporter animals. As control mice, wild-type C57BL/6 mice were used from the animal breeding unit of the Microbiology and Tumor Biology Center (MTC), Karolinska, Stockholm or purchased from Charles River (Sulzfeld, Germany).

Cell isolation and immunostaining. Highly enriched (>98\% E-cadherin ${ }^{+}, \mathrm{CD}^{-} 5^{-}$) primary intestinal epithelial cells were prepared as described before. ${ }^{57}$ In brief, small intestinal tissue was inverted and incubated in $0.3 \mathrm{M} \mathrm{EDTA}$ at $37^{\circ} \mathrm{C}$ for $10 \mathrm{~min}$. Subsequently, the epithelium was removed in large cell aggregates by mechanical sharing and separated at $4{ }^{\circ} \mathrm{C}$ from epithelium-associated leukocytes by repeated differential sedimentation at $1 \times \mathrm{g}$. In addition to the intestinal epithelial cells, the co-eluted epithelium-associated cells from $g f p$ knock-in and wild-type animals were also collected and analyzed by flow cytometry. Also, intestinal intraepithelial lymphocytes were isolated as described recently..$^{40}$ The fraction of epithelium-associated cells and the preparation of intraepithelial lymphocytes each contained a lymphocyte population with similar cellular composition as verified by flow cytometry (data not shown). As the latter protocol resulted in a higher yield, this method was used to obtain cells for subsequent in vitro cell culture. In addition, lamina propria cells and colonic intraepithelial lymphocytes were isolated as described before ${ }^{40,58}$ adding a percoll gradient centrifugation step (40 and 70\%) to enrich colonic intraepithelial lymphocytes. Primary cells were analyzed for GFP expression and co-stained against CD3, CD $8 \alpha$, CD $8 \beta$, CD 4 , TCR $\alpha \beta$, TCR $\gamma \delta$, Foxp3, F4/80, and CD11c following blocking with Mouse BD Fc Block from BD Pharmingen. All flow cytometric analysis was performed using a FACSCalibur (Becton Dickinson, Franklin Lakes, NJ) and analyzed using CellQuest software. Fluorescence-activated cell sorting was performed at the Flow cytometry core facility of the Hanover Medical School and of the MTC, Karolinska Institute. For immunohistology, formalin-fixed small intestinal tissue sections from wild-type and GFP reporter mice were stained using a rabbit anti-GFP antibody followed by detection with a Texas red-conjugated anti-rabbit secondary antibody (Jackson Immunoresearch, Suffolk, England). Immunofluorescence was visualized using a Zeiss Apotomeequipped fluorescence microscope (Jena, Germany).

Stimulation assays. Intestinal epithelial $\mathrm{m}-\mathrm{IC}_{\mathrm{cl} 2}$ cells were cultured as previously described. ${ }^{59}$ For all stimulation experiments, peptidoglycan isolated from Staphylococcus aureus was used at $10 \mu \mathrm{g} \mathrm{ml}^{-1}$ concentration and added apically to polarized epithelial cells in the absence of any transfection reagent. Cells were stimulated for $6 \mathrm{~h}$ if not stated otherwise. Collected cell culture supernatants were stored at $-20^{\circ} \mathrm{C}$ and macrophage inflammatory protein- 2 was quantified using a commercial enzyme-linked immunosorbent assay from Nordic Biosite (Täby, Sweden). Specific or control siRNA was transfected with INTERFERin at a final concentration of $10 \mathrm{nM}$ and $48 \mathrm{~h}$ before functional analysis. Isolated intraepithelial lymphocytes were cultured at $37^{\circ} \mathrm{C}, 5 \% \mathrm{CO}_{2}$ for $18 \mathrm{~h}$ in RPMI-1640 supplemented with $10 \%$ fetal calf serum, 2 mM L-glutamine, 
$100 \mathrm{U}$ penicillin/streptomycin, and $10 \mathrm{~mm}$ HEPES (cell culture medium) and containing $10 \mathrm{ng} \mathrm{ml}^{-1} \mathrm{LPS}, 50 \mathrm{ng} \mathrm{ml}^{-1} \mathrm{IL}-15$, or $50 \mathrm{ng} \mathrm{ml}^{-1} \mathrm{IL}-22$. $\mathrm{Co}$ (control) indicates supplemented cell culture medium without any stimulus. mRNA isolation was performed following the same protocol as for sorted cells described above. Activation assay was performed with isolated intraepithelial lymphocytes. Intraepithelial lymphocytes were cultured in cell culture medium only or in the presence of peptidoglycan or $10 \mathrm{ng} \mathrm{ml}^{-1}$ phorbol myristate acetate and $500 \mathrm{ng} \mathrm{ml}^{-1}$ ionomycin. After $2 \mathrm{~h}$ of stimulation, $10 \mu \mathrm{g} \mathrm{ml}^{-1}$ Brefeldin A was added and intraepithelial lymphocytes were analyzed after an additional $2 \mathrm{~h}$. Immunostaining of $\mathrm{CD} 3$ and intracellular IFN $\gamma$ was performed using the staining buffer set from eBioscience according to the manufacturer's recommendations. Intraepithelial lymphocytes were gated on CD3 and analyzed for IFN $\gamma$ positive cells.

Microbiota analysis. Animals were co-housed and tissues were obtained from 6 -week-old animals ( $n=4$ per group). Tissues were removed and immediately stored at $-80^{\circ} \mathrm{C}$. Total DNA was extracted and quantified. The quantitative PCR was performed precisely as recently described. ${ }^{41}$ All analyses were performed separately for each location (colon, distal, and proximal small intestine). The main analysis tool was GEE-based modeling with robust variance estimates that accounts for the withinanimal correlation of counts of different bacterial classes. For significance testing, only the pre-planned pairwise comparisons of the three groups for each bacterial class were considered. The family-wise type I error rate was controlled at $5 \%$ for each location using the single-step adjustment method based on the multivariate normal distribution. All analyses were performed using 2.10.1 (R Foundation for Statistical Computing, Vienna, Austria) with the geepack 1.0-17 and multcomp 1.1-6 packages.

Statistics. Results represent at least three to four similar experiments and are expressed as mean \pm s.d. The data were analyzed using the unpaired Student's $t$-test. $P<0.05$ was considered significant.

SUPPLEMENTARY MATERIAL is linked to the online version of the paper at http://www.nature.com/mi

\section{ACKNOWLEDGMENTS}

We acknowledge stimulating discussions of our work and technical help from Anders Folkesson (Department of Systems Biology, Technical University of Denmark, Copenhagen), Jochen Hühn, Sascha Cording, Dunja Bruder, Andreas Jeroen (Helmholtz Centre for Infection Research, Braunschweig, Germany), Magdalena Kocur (University of Düsseldorf, Germany), and Jennifer Zaksheske (Medical College of Wisconsin, Milwaukee, WI). Our work was supported by the International Research Training Group 1273 (to C.U.D.) and an individual research grant (to M.W.H.) from the German Research Foundation (DFG), as well as support from the Thyssen Foundation, the Collaborative Research Center SFB 621 and SFB 900, and the German Ministry of Education and Research (DLR 01 GU0825 and 01KI0752). N.H.S. was supported by the NIH grant Al057757 and R.M.L. by the Howard Hughes Medical Institute and the $\mathrm{NIH}$ Al30663.

\section{DISCLOSURE}

The authors declared no conflict of interest.

(c) 2011 Society for Mucosal Immunology

\section{REFERENCES}

1. Inohara, N. et al. Host recognition of bacterial muramyl dipeptide mediated through NOD2. Implications for Crohn's disease. J. Biol. Chem. 278, 5509-5512 (2003).

2. Girardin, S.E. et al. Nod2 is a general sensor of peptidoglycan through muramyl dipeptide (MDP) detection. J. Biol. Chem. 278, 8869-8872 (2003).

3. Franchi, L., Warner, N., Viani, K. \& Nunez, G. Function of Nod-like receptors in microbial recognition and host defense. Immunol. Rev. 227, 106-128 (2009).

4. Kobayashi, K.S. et al. Nod2-dependent regulation of innate and adaptive immunity in the intestinal tract. Science 307, 731-734 (2005).
5. Petnicki-Ocwieja, T. et al. Nod2 is required for the regulation of commensal microbiota in the intestine. Proc. Natl. Acad. Sci. USA 106, 15813-15818 (2009).

6. Hugot, J.P. et al. Association of NOD2 leucine-rich repeat variants with susceptibility to Crohn's disease. Nature 411, 599-603 (2001).

7. Ogura, Y. et al. A frameshift mutation in NOD2 associated with susceptibility to Crohn's disease. Nature 411, 603-606 (2001).

8. Noguchi, E., Homma, Y., Kang, X., Netea, M.G. \& Ma, X. A Crohn's disease-associated NOD2 mutation suppresses transcription of human IL10 by inhibiting activity of the nuclear ribonucleoprotein hnRNP-A1. Nat. Immunol. 10, 471-479 (2009).

9. Dziarski, R. \& Gupta, D. The peptidoglycan recognition proteins (PGRPs). Genome Biol. 7, 232 (2006).

10. Yoshida, H., Kinoshita, K. \& Ashida, M. Purification of a peptidoglycan recognition protein from hemolymph of the silkworm, Bombyx mori. J. Biol. Chem. 271, 13854-13860 (1996).

11. Kang, D., Liu, G., Lundstrom, A., Gelius, E. \& Steiner, H. A peptidoglycan recognition protein in innate immunity conserved from insects to humans. Proc. Natl. Acad. Sci. USA 95, 10078-10082 (1998).

12. Werner, T. et al. A family of peptidoglycan recognition proteins in the fruit fly Drosophila melanogaster. Proc. Natl. Acad. Sci. USA 97, 13772-13777 (2000).

13. Mellroth, P., Karlsson, J. \& Steiner, H. A scavenger function for a Drosophila peptidoglycan recognition protein. J. Biol. Chem. 278, 7059-7064 (2003).

14. Mellroth, P. \& Steiner, H. PGRP-SB1: an N-acetylmuramoyl L-alanine amidase with antibacterial activity. Biochem. Biophys. Res. Commun. 350, 994-999 (2006).

15. Bischoff, V. et al. Downregulation of the Drosophila immune response by peptidoglycan-recognition proteins SC1 and SC2. PLoS Pathog. 2, e14 (2006).

16. Zaidman-Remy, A. et al. The Drosophila amidase PGRP-LB modulates the immune response to bacterial infection. Immunity 24, 463-473 (2006).

17. Liu, C., Gelius, E., Liu, G., Steiner, H. \& Dziarski, R. Mammalian peptidoglycan recognition protein binds peptidoglycan with high affinity, is expressed in neutrophils, and inhibits bacterial growth. J. Biol. Chem. 275, 24490-24499 (2000).

18. Liu, C., Xu, Z., Gupta, D. \& Dziarski, R. Peptidoglycan recognition proteins: a novel family of four human innate immunity pattern recognition molecules. J. Biol. Chem. 276, 34686-34694 (2001).

19. Uehara, A. et al. Chemically synthesized pathogen-associated molecular patterns increase the expression of peptidoglycan recognition proteins via toll-like receptors, NOD1 and NOD2 in human oral epithelial cells. Cell Microbiol. 7, 675-686 (2005).

20. Wang, M. et al. Human peptidoglycan recognition proteins require zinc to kill both gram-positive and gram-negative bacteria and are synergistic with antibacterial peptides. J. Immunol. 178, 3116-3125 (2007).

21. Gelius, E., Persson, C., Karlsson, J. \& Steiner, H. A mammalian peptidoglycan recognition protein with $\mathrm{N}$-acetylmuramoyl-L-alanine amidase activity. Biochem. Biophys. Res. Commun. 306, 988-994 (2003).

22. Wang, Z.M. et al. Human peptidoglycan recognition protein- $L$ is an $N$ acetylmuramoyl-L-alanine amidase. J. Biol. Chem. 278, 49044-49052 (2003).

23. Ladesic, B., Tomasic, J., Kveder, S. \& Hrsak, I. The metabolic fate of 14C-labeled immunoadjuvant peptidoglycan monomer. II. In vitro studies. Biochim. Biophys. Acta. 678, 12-17 (1981).

24. Zhang, Y. et al. Identification of serum $\mathrm{N}$-acetylmuramoyl-I-alanine amidase as liver peptidoglycan recognition protein 2. Biochim. Biophys. Acta. 1752, 34-46 (2005).

25. Hoijer, M.A., Melief, M.J., Debets, R. \& Hazenberg, M.P. Inflammatory properties of peptidoglycan are decreased after degradation by human $\mathrm{N}$ acetylmuramyl-L-alanine amidase. Eur. Cytokine Netw. 8, 375-381 (1997).

26. Xu, M., Wang, Z. \& Locksley, R.M. Innate immune responses in peptidoglycan recognition protein L-deficient mice. Mol. Cell Biol. 24, 7949-7957 (2004).

27. Austyn, J.M. \& Gordon, S. F4/80, a monoclonal antibody directed specifically against the mouse macrophage. Eur. J. Immunol. 11, 805-815 (1981).

28. Schaller, E. et al. Inactivation of the $F 4 / 80$ glycoprotein in the mouse germ line. Mol. Cell Biol. 22, 8035-8043 (2002).

29. Fleming, T.J., Fleming, M.L. \& Malek, T.R. Selective expression of Ly-6G on myeloid lineage cells in mouse bone marrow. RB6-8C5 mAb to granulocyte-differentiation antigen (Gr-1) detects members of the Ly-6 family. J. Immunol. 151, 2399-2408 (1993). 
30. Huleatt, J.W. \& Lefrancois, L. Antigen-driven induction of CD11c on intestinal intraepithelial lymphocytes and CD8+ T cells in vivo. J. Immunol. 154, 5684-5693 (1995).

31. Huleatt, J.W. \& Lefrancois, L. Beta2 integrins and ICAM-1 are involved in establishment of the intestinal mucosal T cell compartment. Immunity 5, 263-273 (1996).

32. Sancho, D., Gomez, M. \& Sanchez-Madrid, F. CD69 is an immunoregulatory molecule induced following activation. Trends Immunol. 26, 136-140 (2005).

33. Barnes, M.J. \& Powrie, F. Regulatory T cells reinforce intestinal homeostasis. Immunity 31, 401-411 (2009).

34. Izcue, A., Coombes, J.L. \& Powrie, F. Regulatory lymphocytes and intestinal inflammation. Annu. Rev. Immunol. 27, 313-338 (2009).

35. Vinay, D.S. \& Kwon, B.S. Role of 4-1BB in immune responses. Semin. Immunol. 10, 481-489 (1998).

36. Ribot, J.C. et al. CD27 is a thymic determinant of the balance between interferon-gamma- and interleukin 17-producing gammadelta $T$ cell subsets. Nat. Immunol. 10, 427-436 (2009).

37. Ballas, Z.K. \& Rasmussen, W. Lymphokine-activated killer cells. VII. IL-4 induces an NK1.1+CD8 alpha+beta - TCR-alpha beta B220+ lymphokine-activated killer subset. J. Immunol. 150, 17-30 (1993).

38. Lin, T. et al. CD3-CD8+ intestinal intraepithelial lymphocytes (IEL) and the extrathymic development of IEL. Eur. J. Immunol. 24, 1080-1087 (1994).

39. Ishimoto, Y. et al. Age-dependent variation in the proportion and number of intestinal lymphocyte subsets, especially natural killer T cells, doublepositive CD4+ CD8+ cells and B220+ T cells, in mice. Immunology 113, 371-377 (2004)

40. Pabst, O. et al. Cryptopatches and isolated lymphoid follicles: dynamic lymphoid tissues dispensable for the generation of intraepithelial lymphocytes. Eur. J. Immunol. 35, 98-107 (2005).

41. Salzman, N.H. et al. Enteric defensins are essential regulators of intestinal microbial ecology. Nat. Immunol. 11, 76-83 (2010).

42. Bates, J.M., Akerlund, J., Mittge, E. \& Guillemin, K. Intestinal alkaline phosphatase detoxifies lipopolysaccharide and prevents inflammation in zebrafish in response to the gut microbiota. Cell Host Microbe 2, 371-382 (2007).

43. Goldberg, R.F. et al. Intestinal alkaline phosphatase is a gut mucosal defense factor maintained by enteral nutrition. Proc. Natl. Acad. Sci. USA 105, 3551-3556 (2008).

44. Goodman, T. \& Lefrancois, L. Intraepithelial lymphocytes. Anatomical site, not $T$ cell receptor form, dictates phenotype and function. J. Exp. Med. 170, 1569-1581 (1989).

45. Bouskra, D. et al. Lymphoid tissue genesis induced by commensals through NOD1 regulates intestinal homeostasis. Nature 456, 507-510 (2008).
46. Atreya, R. et al. Blockade of interleukin 6 trans signaling suppresses T-cell resistance against apoptosis in chronic intestinal inflammation: evidence in Crohn disease and experimental colitis in vivo. Nat. Med. 6, 583-588 (2000).

47. Clarke, T.B. et al. Recognition of peptidoglycan from the microbiota by Nod1 enhances systemic innate immunity. Nat. Med. 16, 228-231 (2010).

48. Saha, S. et al. Peptidoglycan recognition proteins protect mice from experimental colitis by promoting normal gut flora and preventing induction of interferon-gamma. Cell Host Microbe 8, 147-162 (2010).

49. Stepankova, R. et al. Segmented filamentous bacteria in a defined bacterial cocktail induce intestinal inflammation in SCID mice reconstituted with CD45RB high CD4+ T cells. Inflamm. Bowel Dis. 13, 1202-1211 (2007).

50. Lee, Y.K., Menezes, J.S., Umesaki, Y. \& Mazmanian, S.K. Microbes and Health Sackler Colloquium: proinflammatory T-cell responses to gut microbiota promote experimental autoimmune encephalomyelitis. Proc. Natl. Acad. Sci. USA (2010). doi:10.1073/pnas.1000082107.

51. Saha, S. et al. PGLYRP-2 and Nod2 are both required for peptidoglycaninduced arthritis and local inflammation. Cell Host Microbe 5, 137-150 (2009).

52. Janusz, M.J., Chetty, C., Eisenberg, R.A., Cromartie, W.J. \& Schwab, J.H. Treatment of experimental erosive arthritis in rats by injection of the muralytic enzyme mutanolysin. J. Exp. Med. 160, 1360-1374 (1984).

53. Bleich, A. et al. Genetic dissection of granulomatous enterocolitis and arthritis in the intramural peptidoglycan-polysaccharide-treated rat model of IBD. Inflamm. Bowel Dis. 15, 1794-1802 (2009).

54. Edele, F. et al. Cutting edge: instructive role of peripheral tissue cells in the imprinting of T cell homing receptor patterns. J. Immunol. 181, 3745-3749 (2008).

55. Schjoldager, K.T. et al. Cellular cross talk in the small intestinal mucosa: postnatal lymphocytic immigration elicits a specific epithelial transcriptional response. Am. J. Physiol. Gastrointest. Liver Physiol. 294, G1335-G1343 (2008).

56. Pott, J. et al. Internalization-dependent recognition of Mycobacterium avium ssp. paratuberculosis by intestinal epithelial cells. Cell Microbiol. 11, 1802-1815 (2009).

57. Lotz, M. et al. Postnatal acquisition of endotoxin tolerance in intestinal epithelial cells. J. Exp. Med. 203, 973-984 (2006).

58. Weigmann, B. et al. Isolation and subsequent analysis of murine lamina propria mononuclear cells from colonic tissue. Nat. Protoc. 2, 2307-2311 (2007).

59. Bens, M. et al. Transimmortalized mouse intestinal cells (m-ICc12) that maintain a crypt phenotype. Am. J. Physiol. 270, C1666-C1674 (1996). 